\title{
Globalization of Healthcare
}

Michele Mittelman, RN, MPH; Patrick Hanaway, MD

G lobalization - the increasing transnational circulation of money, goods, people, ideas, and information worldwide-is generally recognized as one of the most powerful forces shaping our current and future history. How is it affecting healthcare, and in that context, what is the purpose and significance of Global Advances in Health and Medicine (GAHM), publisher of this journal? Our goal is not homogenization but rather to provide an opportunity for integration, convergence, and collaboration across cultures. By respecting and conserving the richness and diversity of each new medicine, we embrace globalization.

Globalization is of course not new; it began in the Renaissance and particularly with the 15th- and I6thcentury voyages of exploration by Columbus, Magellan, and others. Since the beginning of time, there have been interactions and exchanges among different peoples and cultures. However, the current magnitude of globalization is unprecedented and yet still expanding rapidly. Fueled by advancement in telecommunication technologies and incentivized by immense economic rewards, this multinational development informs innovation in nearly every society on the planet. Economically, national boundaries are dissolving to give way to single global markets for labor, manufacturing, finance, and service. Now taken to be a "given" at the strategic level in every major field of endeavor, the international exchange of ideas poses operative questions that concern not whether or why but when and how. For the first time in the history of mankind, the inception of a truly global community is a foreseeable, if not inevitable, possibility.

For several reasons, medicine and healthcare are uniquely affected by the trend toward globalization. First, medicine benefits from a truly universal reach, embracing virtually the entire world. There are few industries more essential to humanity, and the common desire for health spans the globe. Second, medicine connects various cultural components, with each society employing a model that speaks to social, political, historical, and religious factors. As applied to medicine, globalization has yielded a "flattening" and "converging" effect in which nations and regions that used to be less developed are rapidly catching up with those that are more advanced.

\section{THE FIRST GLOBAL HEALTHCARE INITIATIVE}

Globalized healthcare stems from an ancient idea. The first recorded global initiative in the history of medicine and healthcare occurred in early 7 th-century
Asia, when on the recommendation of his Chinese and Nepalese wives, the founder of the Tibetan Empire, King Songtsen Gampo (605-650), convened the first recorded international medical conference. He invited physicians from China, Nepal, India, Kashmir, and Persia (Iran) to traverse the Silk Road to the Tibetan Plateau, the "Roof of the World," and to bring with them medical texts from their various traditions to be translated into Tibetan for syncretistic study. ${ }^{\mathrm{I}}$

This initial foray into multinational healthcare was so successful that subsequent 8th-century King Trisong-Detson (Gampo's descendant) organized a second multicultural medical conference. Physicians were invited from China, Nepal, India, Kashmir, Persia, and the Turkic regions of central Asia (including what is now Afghanistan) to a second multicultural medical conference. Again, each attendant was obliged to provide at least one medical text for translation and discussion. ${ }^{\mathrm{I}}$ The proceedings were synthesized in a single great book, the Gyu-zhi ("The Four Medical Tantras"), which became the foundation of all subsequent Tibetan Buddhist medicine and is still studied today. Interesting to note, in 2008 and $201 \mathrm{I}$, the Men-TseeKhang, the Tibetan Medical Astrological Institute whose purpose is to preserve and promote Tibetan Medicine, published the first English translation of the first 3 tantras of the Gyu-zhi. Tibetan medicine has become globalized, and as a result, local practices of healing are being transformed in both Tibet and the West. Tibetan medicine is produced as a global commodity and consumed as a "local" tradition.

\section{THE WORLD OF MEDICINE}

The world of medicine is as diverse as the people who have benefitted from it. There exist as many healing systems as there are cultures in the world. Following are descriptions of a few traditional systems (in addition to modern Western American/European practice):

- Chinese medicine, established more than 5000 years ago, is the oldest, encompasses many different practices including acupuncture and herbal remedies, is rooted in the ancient philosophy of Taoism and life energy (known as qi), and is currently practiced together with Western medicine ${ }^{2}$;

- Japanese medicine (Kampo) spun off from the Chinese system in the 7 th century, was developed through empirical trials of herbal medicine and employs more than I40 standardized, regulated, ancient multiherb formulas that are widely
Author Affiliations Michele Mittelman $\mathrm{RN}, \mathrm{MPH}$, is one of the editors of Global Advances in Health and Medicine. Patrick Hanaway, MD, is the cofounder of Family to Family, Asheville, North Carolina; the chief medical officer of Genova Diagnostics, Asheville; and past president of the American Board of Integrative Holistic Medicine.

Correspondence Michele Mittelman, RN, MPH

mmittelman@gahmllc.com

Citation

Global Adv Health Med 2012;1(2):5-7.

\section{Key Words}

Globalization, medicine, healthcare, convergence, Chinese, Japanese, Tibetan, Ayurveda, traditional, holistic 
prescribed and covered by Japan's national healthcare system 3,4 ;

- Tibetan medicine, a blend of many traditions involving the use of herbal remedies, is not officially recognized as a health system but nonetheless is widely practiced from Asia to the Middle East and increasingly in the United States and Europe;

- Korean medicine (Koryo) evolved using Chinese medical concepts, encompasses an exceptionally rich flora, and is practiced together with Western medicine and researched for scientific validations today5;

- Bhutanese medicine (gSo-ba-rig-pa), another ancient tradition with roots in Buddhism and Tibetan traditional medicine, utilizes 3000 species of plants and is practiced in tandem with modern medicine ${ }^{6,7}$;

- Ayurveda in India is based on a strong belief in life energy, uses 2000 plant species, and was founded on the concept that a single consciousness connects everything in the universe ${ }^{8}$;

- Siddha, also practiced in India and similarly based on imbalance of doshas or humors, uses an elaborate and unique diagnostic technique and is integrated into the national health care system 7 ; and

- Unani, a Muslim-Hindu hybrid primarily influenced by Greek, Persian, and Islamic medicine, uses a variety of diets and drugs and is also part of the national health care systems of Greece, Iran, and India. ${ }^{9}$

This list represents only a small fraction of healing techniques currently and historically employed around the world. According to the World Health Organization, about $80 \%$ of people who live in developing countries depend upon sustainable, traditional healing systems

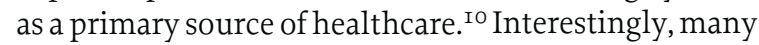
of these healthcare models are not officially recognized or regulated, and some are even prohibited. In Africa, only $6 \mathrm{I} \%$ of nations have legal statutes for traditional medicine systems, and appropriate budgeting for them is usually inadequate or totally lacking. ${ }^{7}$

All of these non-Western systems are holistic; they connect patients' physical symptoms with all other dimensions of their lives. These systems are founded upon the belief that well-being is intrinsically linked to the whole person-body, mind, and spirit-including behavioral, psychological, spiritual, environmental, and cultural factors. The patient is intensely personal, inseparable, and whole. Whereas Western medicine tends to focus on curing illness in various parts of the body, frequently relying upon costly technology that is unaffordable elsewhere, other traditions emphasize a continuum of care. The continuum relies upon preventive medicine, health maintenance, local plants as medicine, food as medicine, and long-term relationships with healers as being far more cost effective.

Interestingly, Western medical science departed alone from the universal healthcare tradition but did so for historical and political — not scientific - reasons. The scientific revolution in the $\mathrm{I} 6 \mathrm{th}$ and $\mathrm{I} 7 \mathrm{th}$ centuries coincided with the religious wars of the Reformation and Counter-Reformation, the dangers of which encouraged scientists to become exclusively specialized, focusing on one safe subject at a time and excluding all others. Though this strategy certainly accelerated the development of Western science and technology, especially when paired with commercial enterprise, it has become increasingly clear that the excluded factors often reemerge as problems.

\section{WHAT LIES AHEAD?}

As modern healthcare practitioners, we should embrace globalization. Technology has eliminated or greatly reduced many of the barriers to communication including distance, time, and cost. The time is right to begin the development of an integrated system that promotes and facilitates the globalization of medicine and healthcare. The Silk Road and the Roof of the World are now in cyberspace, only a click away. The revolution in information technology - global, wireless, and instantaneous communication from human to human, human to machine, and even machine to machine-promotes not only a sense of flattening in medicine and healthcare but also an all-important convergence. At the same time, we do not seek-and in fact actively want to avoid-a drive toward homogenization. Our goal is not to develop a single mechanically enforced and theoretically coherent system. Rather, we must strive for universal integration, constructive convergence, and diverse collaboration that bridges both systems and traditions. We must commit to respect and conserve the richness of each variety of medicine, to ensure complementarity, and to promote creative and mutually enriching hybridization.

The burgeoning awareness of systems biology is encouraging more open-minded and multidisciplinary approaches to problem solving. Complex diseases have called for complex integrative approaches, leading to a new acceptance of outside traditions. Biomedicine is now encouraging us to accept holistic, low-tech, and integrative medical practices as viable options. For the first time in many years, technology has allowed healthcare to re-globalize. With the dissolution of multinational boundaries and the universal free access to information, the notion of holistic and global-based care is emerging as the future of medicine.

It is now generally agreed upon that we have much to learn from our international colleagues, as we all have more in common than we realize. GAHM is a part of this trend and serves as a logical next step in the process. Through the development of an open, interactive, and rapidly expanding communication platform and network, GAHM aims to further accelerate and embody the constructive convergence of advances in health and medicine for the betterment of health management, healthcare delivery, and patient outcomes. GAHM sees the various different approaches to medicine not as mutually exclusive but as complementary and mutually reinforcing. Discussion of various 
healthcare models is conducive to everyone's benefit and ultimately will improve health worldwide. GAHM's focus on case reports will be especially valuable in integrating diverse approaches and therapies at the practical level.

As members of the editorial team of this new journal, we believe that the enormous potential benefits in global convergence and pluralism in medicine and healthcare-in science, in professional practice, and in the basic health of the peoples of the world-are so palpable and exciting that we see GAHM as both a calling and mission. Together, we will learn how to coordinate and synthesize, without homogenization, the wonderfully rich diversity of perspectives for improving the health of all peoples around the globe.

\section{REFERENCES}

I. Clifford T. Tibetan Buddhist medicine and psychiatry: the diamond healing. York Beach (ME): Samuel Weiser, Inc; I984.

2. Traditional Chinese medicine: an introduction. National Center for Complementary and Alternative Medicine website. http://nccam.nih.gov/ health/whatiscam/chinesemed.htm. Accessed April 3, 20 I2.

3. Watanabe K, Matsuura K, Gao P, et al. Traditional Japanese Kampo medicine: clinical research between modernity and traditional medicine - the state of research and methodological suggestions for the future. Evid Based Complement Alternat Med. 20 I I;20 I I:513842.

4. Yamada H. Japanese Kampo medicine. In: Kayne SB, editor. Traditional medi- cine: a global perspective. Philadelphia, PA: Pharmaceutical Press; 20IO. p. 225-7. 5. Sop CT. Koryo system of medicine in DPR Korea. In: Chaudhury RR, Rafei UM, editors. Traditional medicine in Asia. New Delhi: World Health Organization; 2002. p. 69-74.

6. Wangchuk P. Health impacts of traditional medicines and bio-prospecting: a world scenario accentuating Bhutan's perspective. J Bhutan Stud. 20 I I Sept I5.

7. World Health Organization. Legal status of traditional medicine and complementary/alternative medicine: a worldwide review. Geneva: WHO; $200 I$.

8. Kala CP. Current status of medicine plants used by traditional Vaidyas in Uttaranchal State of India. Ethnobot Res Appl. 2005;3:267-78.

9. Saad B, Said O. Greco-Arab and Islamic herbal medicine: traditional system, ethics, safety, efficacy, and regulatory issues. Hoboken (NJ): John Wiley \& Sons, Inc; 201 I.

Io. World Health Organization, World Conservation Union, World Wide Fund for Nature. Guidelines on the conservation of medicinal plants. Gland (Switzerland): WHO, IUCN, WWF; I993. 